\title{
American College of Surgeons Session on Genitourinary Reconstructive Surgery New Orleans 14. Oktober 1992
}

\author{
J. Fichtner \\ Department of Urology, Stanford University, USA
}

Das Meeting, welches sich dieses Jahr den Harnröhrenstrikturen widmete, wurde von J. McCraw eröffnet und begann mit einem interessanten Vortrag von L. Baskin aus Philadelphia über die Genese von Harnröhrenstrikturen.

Die Beobachtung, daß sich Strikturen teilweise erst Jahre nach dem initialen Trauma manifestieren, hat folgende Hypothese zur Entstehung von Strikturen hervorgebracht, welche dann auch im Experiment mit Zellkulturen nachvollzogen wurde. Das initiale Trauma (Katheterismus, Fahrradsturz, etc.) führt zu einer kleinen Narbe in der Urethra, welche bei Miktion einen Druckgradienten aufbaut. Dieser Druckgradient führt zu "stretch" bzw. Dehnung der urethralen Fibroblasten, welche daraufhin vermehrt Kollagen (Typ I und III) bilden und so den Narbungsproze $\beta$ weiter nach proximal stimulieren. Für die Klinik könnte dies bedeuten, daß eine offene Exzision der Striktur die beste Therapie bedeutet, da hiermit die treibende Kraft der Kollagensynthese (initiale Narbe mit Dehnung) entfernt wird.

G. Webster (Durham) wies in einem Übersichtsreferat auf die schlechten Ergebnisse des Sachse in der proximalen Urethra hin, da dort nur wenig spongiöses Gewebe die Harnröhre umgibt und somit nur ein geringer Erfolg durch die Schlitzung erzielt werden kann. Er favorisiert prinzipiell den "pedicle island preputial skin onlay" (PIPS). Im Bereich der bulbären Harnröhre empfiehlt er bei Strikturen unter $1 \mathrm{~cm}$ Länge eine End-zu-End-Anastomose, bei längeren Strikturen island flaps, wobei er, soweit möglich, immer versucht, onlay-flaps zu verwenden, um die Kontinuität der Harnröhre zu wahren.

C. Devine (Norfolk) berichtete über seine Erfahrungen mit Vollhauttransplantaten (Full Thickness Skin Graft-FTSG), die eine geringere Kontraktion als Spalthaut aufweisen, da durch Belassen der Dermis meistens eine bessere Durchblutung gewährleistet ist. Hierfür ist allerdings ein gut vaskularisiertes Bett nötig, welches in der proximalen Harnröhre (wenig spongiosum) nicht vorhanden ist. Wo immer möglich, sollten in diesem Bereich gestielte Lappen als onlay aufgrund der besseren Blutversorgung verwendet werden. Er drainiert seine $\mathrm{Pa}$ -

Akt. Urol. 24 (1993) 165

(c) Georg Thieme Verlag Stuttgart · New York tienten prinzipiell nur über einen Zystofix und verwendet einen Silikonkatheter als HR-stent.

F. Schreiter stellte seine Serie der Spalthauttransplantate (Split Thickness Skin Graft - STSG) bei 51 Patienten vor. Entnahmestelle ist der Oberschenkel, 16 Patienten wiesen Komplikationen (Fistel, Schrumpfung) auf.

J. Duckett (Philadelphia) stellte daraufhin erneut die Mundschleimhaut als Lösung der Probleme und Methode der Wahl vor (die Idee sei ihm aufgrund des Artikels von Houby u. Mitarb. [1941] gekommen). Penishaut sei als Flap mit Blutversorgung gut, als graft nicht. Blasenmukosa ist nicht zuletzt aufgrund der auf der diesjährigen AAP-Tagung von Ransley vorgestellten Serie mit $61 \%$ Komplikationen (Fistel, Striktur, Mukosaprolaps) nicht zu favorisieren. Die histologischen Untersuchungen in Philadelphia zeigten, daß die Mundschleimhaut das dickste Epithel (Stabilität) und die dünnste Lamina propria (leichte Revaskularisierung) aufweist. Blasenmukosa und Penishaut hingegen haben eine dickere Lamina propria und ein dünneres Epithel. Seine Entnahmestelle ist in der Regel die Wange, wenn ein ganzes Rohr benötigt wird, im Falle eines geplanten onlays die Unterlippe.

McAninch (San Francisco) berichtete über 20 Patienten, bei denen er in der proximalen Urethra einen Preputial Skin Circumferential Island Flap vorgenommen hat, wobei er die Haut zusammen mit der DartosFaszie aufgrund der besseren Blutversorgung verwendet. In der Diskussion wurde übereinstimmend betont, daß derzeit die Pedicle Island Penile Skin (PIPS) Flaps die besten Ergebnisse ergeben, wobei hierbei verschiedene Gefäßstiele möglich sind (z. B. Lateral PIPS = Orandi). Scrotal Skin Flaps hingegen zeigen aufgrund von Steinbildung, Divertikeln und Haarwuchs schlechtere Ergebnisse. Free grafts sind nur im Perineum zu empfehlen, da nur dort eine ausreichende Blutversorgung gewährleistet erscheint.

\section{Dr. J. Fichtner}

Department of Urology

Stanford University

USA 